\title{
A Bayesian Test for Equality of Scale Parameters of Several Exponential Distributions
}

\section{L.O. Odongo ${ }^{1}$ and $M$. Samanta ${ }^{2}$}

${ }^{1}$ Department of Mathematics, Kenyatta University, P.O. Box 43844, Nairobi, KENYA; ${ }^{2}$ Department of Statistics, University of Manitoba, Winnipeg, Manitoba, R3T 2N2, CANADA

\section{ABSTRACT}

This article develops a Bayesian test for equality of scale parameters of several exponential distributions. The null distribution of the test statistic is approximated by the chi-square distribution using heuristic reasoning in conjunction with the Wilson-Hilferty transfomation for the chi-square random variable. The accuracy of the chi-square approximation of the test statistic and the modified likelihood ratio statistic is examined and their powers compared using Monte Carlo simulation. The proposed test is found to be comparable to Bartlett's modified likelihood ratio test in terms of accuracy and power. A numerical example is included to illustrate the applications of these tests.

\section{KEYWORDS}

Exponential distribution, Likelihood ratio test, Bartlett's approximation, Bayesian test

\subsection{INTRODUCTION}

In this paper we consider the problem of testing the equality of scale parameters of several two-parameter exponential distributions. The probability density function (pdf) of an exponential distribution with location parameter $\theta$ and scale parameter $\beta$ is given by

$$
f(x ; \theta, \beta)=\frac{1}{\beta} \exp \left\{-\frac{1}{\beta}(x-\theta)\right\}
$$

Where: $\infty<\theta<\infty$ and $0<\beta<\infty$. Suppose $X_{i 1}<X_{i 2}<\ldots<X_{i r i}$ denote the first $r_{i}$ ordered observations in a random sample of size $n_{i}$ from an exponential distribution having the $\operatorname{pdf} f\left(x ; \theta_{i}, \beta_{i}\right), i=1,2, \ldots, k$ where $f(x ; \theta, \beta)$ is as given in equation 1 . We also assume that these $k$ samples are independent of each other. The joint pdf of the $r_{i}$ smallest sample observations $\mathrm{X}_{\mathrm{i} 1}<\mathrm{X}_{\mathrm{i} 2}<\ldots<\mathrm{X}_{\mathrm{ir}}$, is given by 


$$
\begin{aligned}
& \prod_{i=1}^{k} g\left(X_{i 1}, X_{i 2}, \ldots, X_{i r_{i}} ; \theta_{i}, \beta_{i}\right) \\
& =\prod_{i=1}^{k} \frac{n_{i} !}{\left(n_{i}-r_{i}\right) ! \beta_{i}^{r_{i}}} \exp \left[-\frac{1}{\beta_{i}}\left\{\sum_{j=1}^{r_{i}}\left(X_{i j}-\theta_{i}\right)+\left(n_{i}-r_{i}\right)\left(X_{i r_{i}}-\theta_{i}\right)\right\}\right] \\
& \quad \text { if } \theta_{i}<X_{i 1}<X_{i 2}<\ldots X_{r_{i}}<\infty, i=1,2, \ldots, k
\end{aligned}
$$

(Epstien and Sobel 1954).

The problem is to test the null hypothesis $\mathrm{H}_{0}: \beta_{1}=\beta_{2}=\ldots=\beta_{\mathrm{k}}$ against the alternative $\mathrm{H}_{\mathrm{a}}$ : $\beta_{\mathrm{i}} \neq \beta_{\mathrm{j}}$ for some $\mathrm{i} \neq \mathrm{j}$. We first note that (2) is also the likelihood function of the $\mathrm{k}$ samples. We can see that for any $\beta_{\mathrm{i}}>0$, (2) is maximized for $\theta_{\mathrm{i}}=X_{\mathrm{i} 1}$ which is the largest value $\theta_{\mathrm{i}}$ can take for $\mathrm{i}=1,2, \ldots, \mathrm{k}$. We then obtain the maximum likelihood estimates for the scale parameter under the null hypothesis and in the entire parameter space as

where

$$
\sum_{i=1}^{k} \frac{T_{i}}{r} \text { and } \frac{T_{i}}{r_{i}} \text { respectively }
$$

$$
T_{i}=\sum_{j=1}^{r_{i}}\left(X_{i j}-X_{i 1}\right)+\left(n_{i}-r_{i}\right)\left(X_{i r_{i}}-X_{i 1}\right) \text { and } r=\sum_{i=1}^{k} r_{i}
$$

The likelihood ratio criterion for testing $\mathrm{H}_{0}$ against $\mathrm{H}_{a}$ is then given by

$$
\lambda=\frac{r^{r}}{\prod_{i=1}^{k} r_{i}^{r_{i}}} \cdot \frac{\prod_{i=1}^{k} T_{i}^{r_{i}}}{\left(\sum_{i=1}^{k} T_{i}\right)^{r}}
$$

(See Sukhatme, 1936 in the case of complete samples and Epstein and Tsao, 1953 in the case of censored samples). Since

$$
\frac{2 T_{i}}{\beta_{i}}, i=1,2, \ldots, k
$$

are independently distributed as chi-square random variables with $2\left(\mathrm{r}_{\mathrm{i}}-1\right)$ degrees of freedom, it seems reasonable to consider a modified version of the above test statistic proposed by Bartlett (Lawless, 1982, p. 112-114) and given by 


$$
\lambda^{*}=\frac{(r-k)^{r-k}}{\prod_{i=1}^{k}\left(r_{i}-1\right)^{r_{i}-1}} \cdot \frac{\prod_{i=1}^{k} T_{i}^{r_{i}-1}}{\left(\sum_{i=1}^{k} T_{i}\right)^{r-k}}
$$

An approximate level $\alpha$ test for $\mathrm{H}_{0}$ against $\mathrm{H}_{2}$ rejects $\mathrm{H}_{0}$ if

$\frac{-2 \ln \lambda^{*}}{M}$ exceeds $\chi_{k-1, \alpha}^{2}$ where $M=1+\frac{1}{6(k-1)}\left\{\sum_{i=1}^{k} \frac{1}{\left(r_{i}-1\right)}-\frac{1}{(r-k)}\right\}$ and $\chi_{k-1, \alpha}^{2}$

is the upper $100 \alpha \%$ point of the chi-square distribution with (k-1) degrees of freedom.

In section 2 we develop a test of $\mathrm{H}_{0}$ against $\mathrm{H}_{\mathrm{a}}$ using a Bayesian approach. We show that when $\mathrm{H}_{0}$ is true, the proposed test statistic is approximately distributed as a chisquare random variable with $(\mathrm{k}-1)$ degrees of freedom. In section 3 we examine the accuracy of chi-square, approximation of the null distribution of the test statistics for the two procedures and compare their powers using Monte Carlo simulation. We find that both the Bayesian and the likelihood ratio tests are quite accurate and their powers are comparable. Finally, in section 4 we include a numerical example to illustrate the applications of these tests.

\subsection{BAYESIAN TEST}

We note that great effort and concern that may be devoted to the construction and use of a prior distribution that perfectly characterizes available prior information may not reap substantial benefits in terms of greatly improved inferences from the posterior distribution: when the observations are to be made that are expected to be very informative, De Groot (1970) shows that posterior density derived from a non informative prior density over the parameter space is a close approximation to the posterior density derived from a carefully specified proper prior. This is a consequence of the principle of stable estimation discussed in great details by Savage (1962). To this end we now use a heuristic reasoning to construct a Bayesian test for the null hypothesis of equal scale parameters using a non informative prior.

Multiplying the following non informative prior distribution of $\theta_{i}$ and $\beta_{i}, i=1,2, \ldots, k$ 


$$
\pi\left(\theta_{1}, \theta_{2}, \cdots, \theta_{k} ; \beta_{1}, \beta_{2}, \ldots, \beta_{k}\right) \propto \prod_{i=1}^{k} \frac{1}{\beta_{i}}
$$

by the joint pdf of the available sample observation given in (2) we get the joint posterior pdf of $\theta_{\mathrm{i}}$ and $\beta_{\mathrm{i}}, \mathrm{i}=1,2, \ldots, \mathrm{k}$ except for a constant multiplier. We then get the following joint posterior pdf of $\beta_{i}, i=1,2, \ldots, k$ as

$$
\begin{aligned}
h\left(\beta_{1}, \beta_{2}, \cdots, \beta_{k}\right) & \propto \prod_{i=1}^{k} \frac{1}{\beta_{i}^{r_{i}+1}} \int_{-\infty}^{x_{h}} \exp \left[-\frac{1}{\beta_{i}}\left\{T_{i}-n_{i}\left(x_{i 1}-\theta_{i}\right)\right\}\right] d \theta_{i} \\
& \propto \prod_{i=1}^{k} \frac{1}{\beta_{i}^{r_{i}}} \exp \left\{-\frac{T_{i}}{\beta_{i}}\right\}
\end{aligned}
$$

It now follows that $\beta_{1}, \beta_{2}, \ldots, \beta_{\mathrm{k}}$ are a posteriori independent and $\frac{2 T_{i}}{\beta_{i}},(i=1,2, \ldots, k)$ has a chi-square distribution with $2\left(\mathrm{r}_{\mathrm{i}}-1\right)$ degrees of freedom. Applying the Wilson-Hilferty (1931) transformation to $\frac{2 T_{i}}{\beta_{i}}$ we conclude that $\left\{\frac{T_{i}}{\left(r_{i}-1\right)}\right\}^{1 / 3}$ follows approximately a normal distribution with mean $1-\frac{1}{9\left(r_{i}-1\right)}$ and variance $\frac{1}{9\left(r_{i}-1\right)}$. That is, $\beta_{1}, \beta_{2}, \ldots, \beta_{k}$ are a posteriori independent and $\delta_{i}=\left\{\frac{1}{\beta_{i}}\right\}^{1 / 3}$ has approximately a normal distribution with mean $c_{i}=\left\{1-\frac{1}{9\left(r_{i}-1\right)}\right\}\left\{\frac{r_{i}-1}{T_{i}}\right\}^{1 / 3}$ and variance $d_{i}^{2}=\frac{1}{9 T_{i}^{2 / 3}\left(r_{i}-1\right)^{1 / 3}}$. Therefore, $\sum_{i=1}^{k}\left\{\frac{\delta_{i}-c_{i}}{d_{i}}\right\}^{2}$ has approximately a chi-square distribution with $k$ degrees of freedom. It then follows that

$$
\sum_{i=1}^{k} \frac{\left(\delta_{i}-\delta-c_{i}+c\right)^{2}}{d_{i}^{2}}
$$

where $\delta=\left\{\sum_{i=1}^{k} \frac{\delta_{i}}{d_{i}^{2}}\right\}\left\{\sum_{i=1}^{k} \frac{1}{d_{i}^{2}}\right\}^{-1}$ and $c=\left\{\sum_{i=1}^{k} \frac{c_{i}}{d_{i}^{2}}\right\}\left\{\sum_{i=1}^{k} \frac{1}{d_{i}^{2}}\right\}^{-1}$ is approximately distributed as chi-square random variable with (k-1) degrees of freedom. When the null hypothesis is 
true, the random variable in (7) reduces to the statistic $V=\sum_{i=1}^{k} \frac{\left(c_{i}-c\right)^{2}}{d_{i}^{2}}$ and a Bayesian test of $\mathrm{H}_{0}$ against $\mathrm{H}_{a}$ rejects $\mathrm{H}_{0}$ whenever $\mathrm{V}>\chi_{\mathrm{k}-1, \alpha}^{2}$ where $\chi_{k-1, \alpha}^{2}$ is defined as before.

\subsection{Accuracy of the ChI-Square Approximations and Power Results}

To investigate the accuracy of chi-square approximation of the null distribution of the test statistics of the likelihood ratio and the Bayesian procedure, a Monte Carlo simulation study was undertaken in order to estimate the probabilities of type I error for the two tests. Simulation was carried out in SAS version 6 (SAS Institute, 1990) and run on IBM-Compatible mainframe computer. RANEXP command in SAS was used to generate the exponential samples. Estimates were based on 5000 random samples from each of the exponential distributions with $(\theta, \beta)=(6,2),(9,2),(15,2)$. The nominal levels used in our empirical study were $\alpha=0.1,0.05$ and 0.01 . Table 1 summarizes the estimated probabilities of type I errors for the tests using different combinations of sample sizes and various degrees of censoring.

We note from this table that even when $r_{1}, r_{2}$ and $r_{3}$ are small or extremely inhomogeneous the error in chi-square approximation is minor. For the Bayesian test the absolute difference between the probability of type I error and the nominal level of significance $\alpha$ is about $.0068, .0052$ and .0028 for $\alpha=0.1,0.05$ and 0.01 respectively.

We next compare the two test procedures by investigating the powers for different alternatives. Because the power functions of the two tests are not available as explicit mathematical expressions, we used Monte Carlo simulation to estimate the power for selected sample sizes and the alternatives. Simulation was carried out in the same manner as in the case of accuracy of the chi-square approximations and the estimates were based on 5000 random samples. Table 2 summarizes these results. A study of Table 2 reveals that although the power of Bayesian test is always smaller or equal to that of the Likelihood ratio test, the difference between the two powers is very small, the maximum difference being .0014 . 
Table 1. Accuracy of Bayesian test and likelihood ratio test

\begin{tabular}{|c|c|c|c|c|c|c|c|c|c|c|c|}
\hline \multicolumn{3}{|c|}{$\begin{array}{l}\text { Nominal } \\
\text { E.T.I.P }\end{array}$} & \multicolumn{3}{|c|}{ Nominal } & \multirow[t]{2}{*}{ Nominal } & \multirow{2}{*}{$\begin{array}{l}10 \% \\
\text { E.T.I.P } \\
\text { L.R. }\end{array}$} & \multirow[t]{2}{*}{ level } & \multicolumn{2}{|c|}{$5 \%$ level } & $\%$ leve \\
\hline$\underline{n}_{1}$ & $\underline{\mathrm{n}}_{2}$ & $\underline{\mathbf{n}_{3}}$ & $\underline{\mathbf{r}}_{1}$ & $\underline{\mathrm{r}_{2}}$ & $\underline{\mathbf{r}}_{3}$ & & & & $\underline{\mathrm{L}, \mathrm{R}}$. & & $\underline{\text { L.R.R. }}$ \\
\hline 15 & 15 & 15 & 4 & 4 & 4 & .0946 & .0988 & .0490 & .0532 & .0992 & .0120 \\
\hline 15 & 15 & 15 & 6 & 6 & 6 & .0966 & .0980 & .0478 & .0494 & .0090 & .0110 \\
\hline 15 & 15 & 15 & 8 & 8 & 8 & .0958 & .0972 & .0482 & .0488 & .0072 & .0078 \\
\hline 15 & 15 & 15 & 10 & 10 & 10 & .0988 & .1000 & .0486 & .0496 & .0080 & .0084 \\
\hline 10 & 10 & 10 & 3 & 5 & 6 & .0934 & .0968 & .0444 & .0514 & .0100 & .0118 \\
\hline 10 & 15 & 20 & 5 & 8 & 10 & .0964 & .0980 & .0498 & .0548 & .0086 & .0082 \\
\hline 10 & 15 & 60 & 3 & 20 & 40 & .0976 & .0972 & .0458 & .0472 & .0078 & .0102 \\
\hline 10 & 60 & 10 & 4 & 40 & 8 & .0968 & .0976 & .0500 & .0544 & .0094 & .0094 \\
\hline 60 & 60 & 60 & 50 & 50 & 50 & .1040 & .1044 & .0540 & .0544 & .0106 & .0106 \\
\hline 10 & 40 & 100 & 5 & 25 & 60 & .1050 & .1034 & .0514 & .0528 & .0100 & .0090 \\
\hline 10 & 50 & 100 & 6 & 30 & 70 & .1006 & .1004 & .0510 & .0514 & .0094 & .0100 \\
\hline 10 & 60 & 100 & 3 & 50 & 70 & .1068 & .1052 & .0476 & .0510 & .0090 & .0100 \\
\hline 10 & 30 & 100 & 4 & 12 & 60 & .0996 & .1002 & .0542 & .0516 & .0112 & .0110 \\
\hline 15 & 60 & 100 & 10 & 40 & 80 & .1032 & .1028 & .0534 & .0518 & .0110 & .0104 \\
\hline
\end{tabular}

E.T.I.P=Estimated Type I Error Probability; Bayes=Bayesian Test, L.R=Likelihood Ratio Test

Table 2. Simulated power $\left(\alpha=0,10, \theta_{1}=6, \theta_{2}=9, \theta_{3}=15, \beta_{1}=2\right)$

\begin{tabular}{|c|c|c|c|c|c|c|}
\hline \multirow[t]{8}{*}{$\frac{\underline{r}_{1}\left(=\mathrm{n}_{1}\right)}{15}$} & $\frac{\mathrm{r}_{2}\left(=\mathrm{n}_{2}\right)}{15}$ & $\frac{\mathrm{r}_{3}\left(=\mathrm{n}_{3}\right)}{15}$ & $\frac{\beta_{2}}{2.0}$ & $\frac{\beta_{3}}{2.0}$ & $\frac{\text { Bayes }}{.1014}$ & $\frac{\mathrm{LR}}{.1024}$ \\
\hline & & & 2.0 & 2.5 & .1562 & .1576 \\
\hline & & & 2.0 & 3.0 & .2840 & .2852 \\
\hline & . & & 2.5 & 3.5 & .3590 & .3602 \\
\hline & & & 2.5 & 4.0 & .4922 & .4932 \\
\hline & & & 3.0 & 3.5 & .3624 & .3638 \\
\hline & & & 3.0 & 4.0 & .4778 & .4790 \\
\hline & & & 5.0 & 6.5 & .8920 & .8928 \\
\hline \multirow[t]{7}{*}{25} & 25 & 25 & 2.0 & 2.0 & . 1070 & .1070 \\
\hline & & & 2.0 & 2.5 & .1946 & .1950 \\
\hline & & & $\begin{array}{l}2.0 \\
2.5\end{array}$ & $\begin{array}{l}3.0 \\
3.5\end{array}$ & $\begin{array}{l}.4042 \\
.5126\end{array}$ & $\begin{array}{l}.4050 \\
.5134\end{array}$ \\
\hline & & & 2.5 & 4.0 & .6982 & .6984 \\
\hline & & & 3.0 & 3.5 & .5152 & .5260 \\
\hline & & & 3.0 & 4.0 & .6772 & .6768 \\
\hline & & & 5.0 & 6.5 & .9896 & .9896 \\
\hline
\end{tabular}

Bayes=Bayesian test, $L R=$ Likelihood ratio test 


\subsection{NUMERICAL EXAMPLE}

For the purpose of illustration we consider three sets of data presented in Bain (1978, p.203). These data relate to the duration of tire tread wear, taken from three methods of production, which are called Present, Additive, and Thickness, respectively. Each set of data represents the first 20 failure times of size 40 . The data are reproduced in Table 3.

Table 3. Tire Failure Data (in thousands of miles)

$\begin{array}{lllllllllll}\text { Present: } & 10.03 & 10.47 & 10.58 & 11.48 & 11.60 & 12.41 & 13.03 & 13.51 & 14.48 & 16.96 \\ & 17.08 & 17.27 & 17.90 & 18.21 & 19.30 & 20.10 & 21.51 & 21.78 & 21.79 & 25.34 \\ \text { Additive: } & 10.10 & 11.01 & 11.20 & 12.95 & 13.19 & 14.81 & 16.03 & 17.01 & 18.96 & 24.10 \\ & 24.15 & 24.52 & 26.05 & 26.44 & 28.59 & 30.24 & 31.03 & 33.51 & 33.61 & 40.68 \\ \text { Thickness: } & 19.07 & 19.51 & 19.62 & 20.47 & 20.78 & 21.37 & 22.08 & 22.61 & 23.47 & 26.02 \\ & 26.23 & 26.47 & 27.07 & 27.43 & 28.28 & 29.10 & 29.66 & 30.67 & 30.81 & 34.36\end{array}$

Data taken from Bain (1978, p.203)

For these data, we have $k=3, n_{1}=n_{2}=n_{3}=40, r_{1}=r_{2}=r_{3}=20, M=1.01, T_{1}=429.43, T_{2}=857.78$ and $T_{3}=429.48$. We obtain $\frac{-2 \ln \lambda^{*}}{M}=6.36$ and from the chi-square tables we have $\chi_{2, .05}^{2}=5.99$. Hence, the likelihood ratio test rejects the null hypothesis of equal scale parameters at 5\% level of significance. Similarly, the value of the Bayesian test statistic calculated from the above data set is $V=6.34$ and the null hypothesis of equal scale parameters is rejected at the same level.

\section{ACKNOWLEDGEMENTS}

We thank the referees for very helpful comments on the paper.

\section{REFERENCES}

Bain L.J. (1978) Statistical Analysis of Reliability and Life-Testing Models. New York: Marcel Dekker.

DeGroot M.H. (1970) Optimal Statistical Decisions. New York: McGraw-Hill. Epstein B. and M. Sobel (1954) Some Theorems Relevant to Life testing From an Exponential Distribution. Annals of Mathematical Statistics 25, 373-381. 
Epstein B. and C.K. Tsao (1953) Some Tests Based on Ordered Observations From Two Exponential Populations. Annals of Mainematical Statistics 24, 456-466.

Lawless J.F. (1982) Statistical Models and Methods for Lifetime Data. New York: John Wiley.

SAS Institute Inc. (1990) SAS language: Reference, Version 6, First Edition Cary NC: Author.

Savage L.J. (1962) The Foundations of Statistical Inference. London: Methuen. Sukhatme P.V. (1936) On Analysis of k Samples From Exponential Population With Special References to the Problem of Random Intervals Statistical Research Memoirs 94, 112.

Wilson E.B. and M.M. Hilferty (1931) The Distribution of Chi-square Proc. Nat. Academy of Sci. 17, 684-688. 